\title{
UMS CHAU
}

\section{0 jähriges Firmenjubiläum von Dr. Walter Kling}

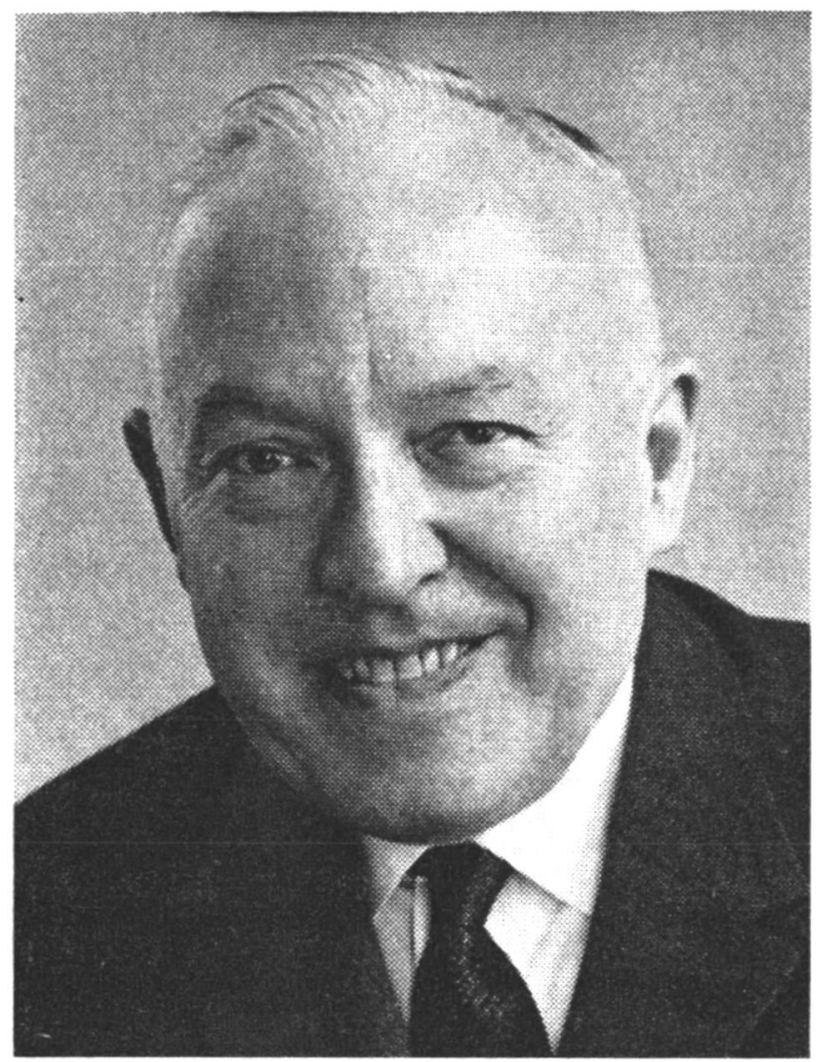

Am 1. Oktober 1966 kann Dr. Walter Kling auf eine 40 jährige Arbeitszeit bei den Firmen Böhme und Henkel zurücksehen. Nach seinem Studium an der Universität Innsbruck (Dissertation 1925 bei Professor K. BRUNNeR über Indolinone) trat er 1926 in die $H$. Th. Böhme $A G$, Chemnitz, ein, in der er schließlich 1943, nach der Übersiedlung von H. BERTSCH nach Berlin bzw. Rodleben, die Leitung des gesamten Chemiebereichs des Chemnitzer Werkes übernahm. 1946-1962 gehörte er der im Westen neu gegründeten Böhme Fettchemie GmbH an, deren stellvertretender Geschäftsführer er 1956 wurde und die 1962 von der Henkel \& Cie. GmbH, Düsseldorf übernommen wurde. Ebenfalls 1956 übernahm W. KLING jedoch schon die Leitung der Anwendungstechnik V der Henkel \& Cie. GmbH. Hier wurden die physikochemischen Grundlagen des Waschvorgangs erforscht und die sich daraus ergebenden theoretischen und experimentellen Ergebnisse bei der Herstellung neuer Produkte, insbesondere Feinwasch- und Spülmittel, ausgewertet. Hinzu kamen später die Nahrungsmittel-Laboratorien, die Abteilung für Elektronenmikroskopie und Photographie und das neu errichtete toxikologische Laboratorium. Heute leitet W. KLING die gesamte Markenartikelentwicklung der Henkel-Gruppe.

Viele der Arbeiten von W. KLING befaßten sich mit der Entwicklung neuer Wasch-, Textil- und Lederhilfsmittel. Zahlreiche wissenschaftliche Publikationen sind in diesen Jahren entstanden, von denen nur einige Themen angeführt werden sollen: Ablösung öliger Verschmutzungen, Umnetzungsvorgänge, Berechnung von Wascharbeiten, elektrische Phänomene bei Faser und Schmutz, elektronenmikroskopische Untersuchungen über die Morphologie von Fasern, Entfernung von Schmutzpigmenten, Kristallisations- und Fibrillenbildungsvermögen von Tensiden, Messung von Haftkräften und Abhängigkeit der Haftkräfte von Wasser und wäßrigen Tensidlösungen, Untersuchungen über Hautverträglichkeit von Waschmitteln und zur allgemeinen Toxikologie von Wasch- und Spülmitteln, Bearbeitung von Abwasserfragen im Zusammenhang mit dem Detergentiengesetz u.a.m.

W. KLING ist Mitarbeiter zahlreicher amtlicher Stellen, Verbände und Institutionen, so etwa Vorsitzender des Verbandes der Textilhilfsmittel-, Lederhilfsmittel-, Gerbstoff- und Waschrohstoffindustrie (TEGEWA) und als solcher Mitglied des erweiterten Vorstandes des Verbandes der chemischen Industrie $(V C I)$, Vorsitzender des Kuratoriums der Wäschereiforschung Krefeld, Mitglied der Kuratorien der Forschungsstelle Chemischreinigung Krefeld (FCR) und der Textilforschungsanstalt Krefeld (TFA) und Mitglied des Vorstands des Vereins der Textilchemiker und Coloristen (VTCC).

IFAT 1966 und III. Internationale Abwasserkonferenz, München

Die Internationale Fachmesse für Abwassertechnik (IFAT) zeigte vom 3.-9. 9. 1966 in München auf $13500 \mathrm{~m}^{2}$ Hallenfläche und $21000 \mathrm{~m}^{2}$ Freigeländefläche Erzeugnisse von 151 Ausstellern des In- und Auslands und gab einen wichtigen Überblick über den derzeitigen Stand der Abwassertechnik und der angrenzenden Gebiete. Veranstalter der IFAT war die Münchener Messe- und Ausstellungsgesellschaft $\mathrm{mbH}$, ideeller Träger die Abwassertechnische Vereinigung eV, Bonn.

Gleichzeitig mit der IFAT fand vom 5.-9. 9. 1966, ebenfalls auf dem Münchener Messegelände, die III. Internationale Abwasserkonferenz statt, die, als Gast der Abwassertechnischen Vereinigung, von der International Association on Water Pollution Research veranstaltet wurde. Auf dieser Konferenz wurden 48 Fachvorträge von namhaften Fachleuten in drei Gruppen gehalten: Allgemeiner Gewässerschutz, Abwasserbehandlung und Ableitung von Abwasser in das Meer.

Sowohl über die IFAT wie über die für das Tensidgebiet wichtigen Vorträge und Ergebnisse der Abwasserkonferenz wird im kommenden Heft ausführlicher berichtet werden. (Ts 1362)

\section{Tagungen}

Die nächstjährige Hauptversammlung der Max-Planck-Gesellschaft zur Förderung der Wissenschaften e.V. findet vom 7.-9. Juni 1967 in Kiel statt.

Die Internationale Fachtagung Textilreinigung 1966 findet vom 1.-3. November in Magdeburg statt (Fachverband „,TextilBekleidung-Leder" der Kammer der Technik, 108 Berlin, Clara-Zetkin-Straße 115/117; Tagungsbüro: Kronenstr. 18).

Das Internationale Technicon-Symposium Automation in der analytischen Chemie wird vom 2.-4. November 1966 in Paris abgehalten.

\section{Berichtigung}

Einsprüche zum Normen-Entwurf DIN 53911 [Tenside 3 (1966) 340-341] werden bis zum 31. Juli 1967 an den Fachnormenausschuß Materialprüfung, 4600 Dortmund, Hakenstraße 5, erbeten.

\section{Inhalt}

L. Hartmann und H. Mosebach, Adsorption mit neuen Detergentien

H. STACHe, Lagereigenschaftsverbessernde Zusätze für Waschpulver, hergestellt mit geradkettigen Na-Alkylbenzolsulfonaten

H. E. Tschakert, Schaum, ein anwendungstechnisches Problem (2. Teil)

Jahresversammlung des CID-Vorstands, Paris 1966

4. Internationaler Polarographischer Kongreß, Prag 1966
Deutsche Normen (Bestimmung der Einfülldichte von Pasten, Salben und Gallerten).

Patentberichte:

Deutsche Patentanmeldungen . . . . . . . . . . . 368

Auslandspatente . . . . . . . . . . . . . . . . . 374

Zeitschriftenreferate . . . . . . . . . . . . . 375

Buchbesprechungen . . . . . . . . . . . . . . . . . 379

Umschau

Der Verlag behält sich das ausschließliche Recht der Vervielfältigung in jeglicher Form, auch der der fotomechanischen Wiedergabe, vor, jedoch wird gewerblichen Unternehmen für den innerbetrieblichen Gebrauch entsprechend dem zwischen dem Börsenverein des Deutschen Buchhandels und dem Bundesversand der Deutschen Industrie abgeschlossenen Rahmenabkommen die Anfertigung einer fotomechanischen Vervielfältigung gestattet. Werden die Gebühren durch Wertmarken entrichtet, so ist eine Marke im Betrag von DM 0,30 für jedes Fotokopierblatt zu entrichten. Durch den Verlag können Fotokopien zum Preis von DM 1,60 je DIN A 4-Seite bezogen werden. Die Wiedergabe von Gebrauchsnamen, Handelsnamen, Warenbezeichnungen usw. in dieser Zeitschrift berechtigt nicht zu der Annahme, daß solche Namen ohne weiteres von jedermann benützt werden dürfen; oft handelt es sich um gesetzlich geschützte eingetragene Warenzeichen. - TENSIDE erscheint monatlich. Bezugspreis vierteljährlich DM 24,- zuzüglich 60 Pfg. Porto. Berechnung vierteljährlich zu Quartalsbeginn. Abbestelltermin spätestens 1 Monat vor Quartalsschluß. Auslandspreis jährlich DM 98,60 einschließlich Versandkosten.

Verlag: Carl Hanser Zeitschriftenverlag GmbH (Gesellschafter Dr. Carl Hanser), München 27, Kolbergerstr. 22. Anzeigenver waltung: Carl Hanser Zeitschriftenverlag GmbH, München 27, Kolbergerstraße 22. Gerichtsstand: München. Redaktion: Dr.-Ing. Richard E. Huttenlocher und Dipl.-Chem. Th. Steudel, München, Kolbergerstraße 22, Tel. 48 5861, Telex 05-22837. Satz, Druck und Bindearbeiten: Graphische Betriebe Dr. F. P. Datterer \& Cie. Nachf. Sellier OHG, Freising Printed in Germany. 